\section{The analysis of lacquer crack in the assessment of myopic choroidal neovascularization}

YM Kim ${ }^{1,2}$, JU Yoon ${ }^{1,2}$ and HJ Koh ${ }^{1}$

\begin{abstract}
Objective The aim of this study was to clarify the characteristic findings in myopic choroidal neovascularization (CNV) and the relationship with lacquer crack (LC).

Methods In all, 66 consecutive myopic CNV patients treated with photodynamic therapy and/or intravitreal anti-vascular endothelial growth factor injection in one eye were reviewed. Data from fluorescein angiography (FA) and indocyanine green angiography (ICGA), obtained simultaneously using the Heidelberg retina angiograph 2 (HRA2), were analyzed.
\end{abstract}

Results LCs were associated with a relatively large extent ( $\geq 3000 \mu \mathrm{m})$ of peripapillary choroidal atrophy and a dark rim, the proliferation of retinal pigment epithelial cells surrounding the neovascular membrane was accompanied by a small extent. Myopic CNV usually developed in the LC area surrounded by tiny crack fragments. In all, 35 patients with LCs received FA and ICGA at least twice during follow-up. LC progression was observed in nine $(25.7 \%)$ treated eyes and six $(23.1 \%)$ non-CNV fellow eyes. Crack fragments progressed in three distinct forms such as elongation, branching, or bridging pattern. Newly diagnosed myopic CNV was reported in 18 treated eyes and 3 fellow eyes. Progression of LCs and development of CNV occurred simultaneously in eight eyes. By multivariate Cox's regression, a statistically significant association was observed between recurrence of myopic CNV and the absence of a dark rim on ICGA.

Conclusions The HRA2 instrument affords detailed high-resolution images of FA and ICGA. Notably, recurrence of myopic CNV developed in areas surrounded by new small crack fragments and LCs are considered to be important in the development of myopic CNV.
Eye (2011) 25, 937-946; doi:10.1038/eye.2011.94; published online 29 April 2011

Keywords: indocyanine green angiography; lacquer crack; myopic choroidal neovascularization

\section{Introduction}

Pathological myopia is the leading cause of severe visual loss in many countries. ${ }^{1,2}$ High myopia is especially common in Asian populations, with rates of $9-21 \%,{ }^{3,4}$ compared with $2-4 \%$ in Caucasians. ${ }^{5-7}$

The pathogenesis of high myopia is associated with progressive and excessive elongation of the eyeball which predisposes to various degenerative changes involving the sclera, choroid, and retina. ${ }^{8,9}$ Myopic choroidal neovascularization $(\mathrm{CNV})$, the visionthreatening complication of myopia, ${ }^{10,11}$ develops in $5-11 \%$ of patients with high myopia. $8,12,13$

Indocyanine green angiography (ICGA) can provide valuable information on choroidal vessels because of its longer wavelength fluorescence with limited diffusion within the choriocapillaries compared with fluorescein angiography (FA). ${ }^{14-16}$ Many characteristic findings of myopic CNV are observed using ICGA such as lacquer cracks (LCs), peripapillary choroidal atrophy, any dark rim, and late-phase hyperfluorescence. In particular, LCs are considered important in the development of the condition, ${ }^{13,17,18}$ but the associated mechanism remains unclear so far.

The Heidelberg retina angiograph 2 (HRA2; Heidelberg Engineering, Heidelberg, Germany), a confocal scanning system, can yield detailed images and perform FA and ICGA simultaneously allowing comparison of images obtained at the
${ }^{1}$ The Institute of Vision Research, Department of Ophthalmology, Yonsei University College of Medicine, Seoul, Korea

Correspondence: HJ Koh, The Institute of Vision Research, Department of Ophthalmology, Yonsei University College of Medicine, 134 Shinchondong, Seodaemoon-gu, Seoul 120-752, Korea Tel: + 8222228 3573; Fax: + 8223120541

E-mail: hjkoh@yuhs.ac ${ }^{2}$ These authors contributed equally to this work.

Received: 27 September 2010

Accepted in revised form: 9 March 2011

Published online: 29 April 2011

The abstract of this article was presented as an oral presentation at the World Ophthalmology Congress 2010. 
same point of the retina. However, to the best of our knowledge, few reports on the use of this technique in the assessment of myopic CNV patients have appeared.

The purpose of this study was to identify characteristic findings of myopic CNV, especially LC and to describe factors prognostic for recurrence of the condition.

\section{Materials and methods}

\section{Subjects}

We retrospectively reviewed the medical records of 66 consecutive myopic CNV patients who received photodynamic therapy (PDT) and/or intravitreal ranibizumab or bevacizumab injection between 1 March 2005 and 31 May 2008 at the Department of Ophthalmology, Yonsei University College of Medicine, Seoul, Korea. This study was conducted in accordance with the principles of the Declaration of Helsinki and carried out with the approval of our Institutional Review Board. Potential risks and benefits were discussed with all patients before they received treatment and each patient read and signed an informed consent form.

Patients were included if they met all of the following criteria: (1) previous treatment with PDT and/or intravitreal anti-vascular endothelial growth factor (anti-VEGF) antibody (ranibizumab or bevacizumab) for myopic $\mathrm{CNV}$ in one eye, defined as the presence of active leakage from the $\mathrm{CNV}$ on $\mathrm{FA}$, with a refractive error exceeding -6.0 diopters (spherical equivalent) or an axial length of $\geq 26.5 \mathrm{~mm}$; and (2) subfoveal, juxtafoveal, or extrafoveal myopic CNV. Exclusion criteria included (1) a history of laser photocoagulation, radiation, subtenon injection of triamcinolone acetonide, or vitreoretinal surgery on the study eye; (2) absence of FA and ICGA data at baseline; and (3) the presence of diabetic retinopathy, branch retinal vein occlusion, or other vascular diseases that might affect FA and ICGA.

\section{Examination}

Initial evaluation included refraction measurement, fundus photography, FA, ICGA, and optical coherence tomography (OCT). FA and ICGA were performed simultaneously using a confocal laser scanning system (HRA2). The presence and location of each LC were identified in the late phase of ICGA. The presence of LC and progression of LC with development of new CNV during follow-up period was also evaluated in the non-CNV fellow eyes. A dark rim was defined as a circular background hypofluorescence surrounding the neovascular membrane, and was detected in the early phase of ICGA. Late-phase ICGA hyperfluorescence was also noted. The extent of peripapillary choroidal atrophy was measured as the greatest linear dimension (GLD) of the hypofluorescent area around the disc, as seen in the late phase of ICGA. All measurements were taken by a physician masked to patient details.

\section{Treatment}

Patients who received standard PDT were treated using the protocol described in the Verteporfin in Photodynamic Therapy study. In brief, spot size was determined after measuring the GLD of the entire CNV lesion by FA. An additional $1000 \mu \mathrm{m}$ was added to the GLD figure to provide a $500-\mu \mathrm{m}$ treatment rim around the lesion. The dose and timing of verteporfin infusion were $6 \mathrm{mg} / \mathrm{m}^{2}$ body surface area over a 10-min period, and laser light $\left(50 \mathrm{~J} / \mathrm{cm}^{2}\right.$ for $83 \mathrm{~s}$ ) was delivered at $15 \mathrm{~min}$ after the start of infusion.

Intravitreal anti-VEGF injections delivered $1.25 \mathrm{mg}$ of bevacizumab or $0.5 \mathrm{mg}$ of ranibizumab. Drugs were injected into the vitreous cavity through the superior sclera using a 30-gauge needle, at a position $4 \mathrm{~mm}$ posterior to the corneal limbus.

Follow-up visits were arranged at 1 week and at 1 month after the initial treatment, then scheduled at 3-month interval; work-up at these visits included FA and OCT. The treatment was repeated when recurrence of myopic CNV was detected. Recurrence was defined as the CNV leakage observed by FA or subretinal fluid visible on OCT.

\section{Statistical analysis}

To investigate factors contributing to specific findings on ICGA and to compare the morphological appearance of LCs, Student's $t$-tests for analysis of continuous variables and $\chi^{2}$-tests exploring categorical variables were used. Multiple logistic regression and Cox's regression analysis were used to elucidate the independent influence of each potential factor on presence of LCs and recurrence of myopic CNV, respectively. Statistical analysis was performed using SPSS 15.0 for Windows (SPSS Inc., Chicago, IL, USA) and SAS version 9.13 (SAS, Cary, NC, USA). Mauchly's test of sphericity and KolmogorovSmirnov analysis were used to confirm statistical validity.

\section{Results}

\section{Baseline patient characteristics}

The characteristics of the 66 patients (66 eyes) with treatment of myopic CNV in one eye are summarized in Table 1. In all, 12 men (18.2\%) and 54 women (81.8\%) were included in the study. An LC was detected in 37 eyes, of which 22 showed LCs of linear-type and 
Table 1 Baseline characteristics of patients $(n=66)$

\begin{tabular}{lc}
\hline Characteristics & \\
\hline Age & $46.9 \pm 12.1$ \\
Male/total (\%) & $12 / 66(18.2)$ \\
Pseudophakic/total (\%) & $5 / 66(7.6)$ \\
Refractive error (diopters) & $-10.6 \pm 5.4$ \\
GLD of CNV ( $\mu$ m) & $1720 \pm 559$ \\
& \\
Location of CNV & $30(45.4)$ \\
Subfovea (\%) & $30(45.4)$ \\
Juxtafovea (\%) & $6(9.1)$ \\
Extrafovea (\%) & \\
Treatment of CNV & $22(33.3)$ \\
PDT only (\%) & $18(27.3)$ \\
PDT + anti-VEGF injection (\%) & $26(39.4)$ \\
Anti-VEGF injection only (\%) & $0.57 \pm 0.36$ \\
Baseline corrected VA (logMAR)
\end{tabular}

Abbreviations: anti-VEGF, anti-vascular endothelial growth factor; $\mathrm{CNV}$, choroidal neovascularization; GLD, greatest linear dimension; PDT, photodynamic therapy; VA, visual acuity.

Table 2 Comparison of clinical features between subgroups based on the size of peripapillary choroidal atrophy

\begin{tabular}{lccc}
\hline & $\begin{array}{c}<3000 \mu m \\
(\mathrm{n}=33)\end{array}$ & $\begin{array}{c}\geq 3000 \mu m \\
(\mathrm{n}=33)\end{array}$ & P-value \\
\hline $\begin{array}{l}\text { Presence of lacquer } \\
\text { crack (\%) }\end{array}$ & $4 / 33(12.1)$ & $29 / 33(87.9)$ & $<0.001$ \\
$\begin{array}{l}\text { Presence of dark rim (\%) } \\
23 / 33(69.7)\end{array}$ & $10 / 33(30.3)$ & 0.003 \\
\hline
\end{tabular}

The $\chi^{2}$-test was used to compare nominal variables.

15 stellate-type LCs. A dark rim was found in 33 eyes. Late-phase hyperfluorescence was observed in 12 eyes. In the non-CNV fellow eyes, there was an LC of 26 eyes, of which 17 were LCs of linear-type, and 9 were stellate-type.

\section{Relationship between features on ICGA}

The presence of an LC was associated with a relatively large degree $(\geq 3000 \mu \mathrm{m})$ of peripapillary choroidal atrophy $(P<0.001)$. However, myopic $\mathrm{CNV}$ with a dark rim showed a small extent $(<3000 \mu \mathrm{m})$ of such atrophy $(P=0.003$; Table 2). Figure 1 shows instances of myopic $\mathrm{CNV}$ without an LC but with a dark rim around the neovascular membrane, as seen on early phase ICGA.

\section{The location of myopic CNV}

In patients with linear-type LCs, the location of myopic $\mathrm{CNV}$ was either within the area of the LC (11 eyes, 50.0\%) or at the tip of the LC (11 eyes, 50.0\%). However, CNV always occurred in the $\mathrm{LC}$, the location nearest to the fovea and, especially, in a region surrounded by very tiny crack fragments. Therefore, the spatial relationship between LC and fovea was important to detect the site of myopic CNV (Figure 2). In patients with stellate-type LCs, myopic CNV was located near the center of the LC (14 eyes, 93.3\%) or at the tip of the LC (1 eye, 6.7\%). The center of the LC usually did not coincide with the fovea, and $\mathrm{CNV}$ tended to occur in the inner corner of the radiating arm nearest to the fovea. Figure 3 shows instances of myopic $\mathrm{CNV}$ associated with stellatetype LCs.

Myopic CNV did not develop from the precise position of the LC per se. An LC consisted of a collection of small dot-shaped crack fragments and CNV was located in the area surrounded by these fragments, as shown in Figures 2 and 3. Small LC fragments were clearly noted in 25 eyes and the location of CNV corresponded with the region surrounded by such tiny dot-shaped crack fragments in 23 eyes.

\section{Subgroup analysis according to presence of $L C$}

There was no significant difference in age, refractive error, visual acuity, CNV size, extent of peripapillary choroidal atrophy, presence of a dark rim, or location of CNV between the patients with two types of LCs. Table 3 illustrates possible clinical factors affecting the occurrence of an LC. Older age, higher refractive error, absence of a dark rim, and greater extent of peripapillary choroidal atrophy were associated with the presence of an LC. After multivariate logistic regression analysis, only the area of peripapillary choroidal atrophy showed a significant correlation with occurrence of an LC $(P=0.02)$.

\section{Progression of $L C$}

In all, 35 treated eyes and 26 non-CNV fellow eyes with LCs were examined by FA and ICGA more than twice during the follow-up period and selected for analysis of LC progression. The mean time interval between the initial and final ICGA examinations was $17.4 \pm 10.6$ months. LC progression was observed in nine (25.7\%) treated eyes and six $(23.1 \%)$ non-CNV fellow eyes. Progression of LC and recurrence of myopic CNV occurred simultaneously in five treated eyes, and new CNV with progression of LC developed in three non-CNV fellow eyes. In the other four treated and three fellow eyes, LC progression was noted, but was not accompanied by outbreak of CNV.

LC progression was associated with the production of increasing amounts of small crack fragments. These fragments appeared slowly, in three different patterns, arising from the tip of an existing LC (elongation, 66.7\%), 


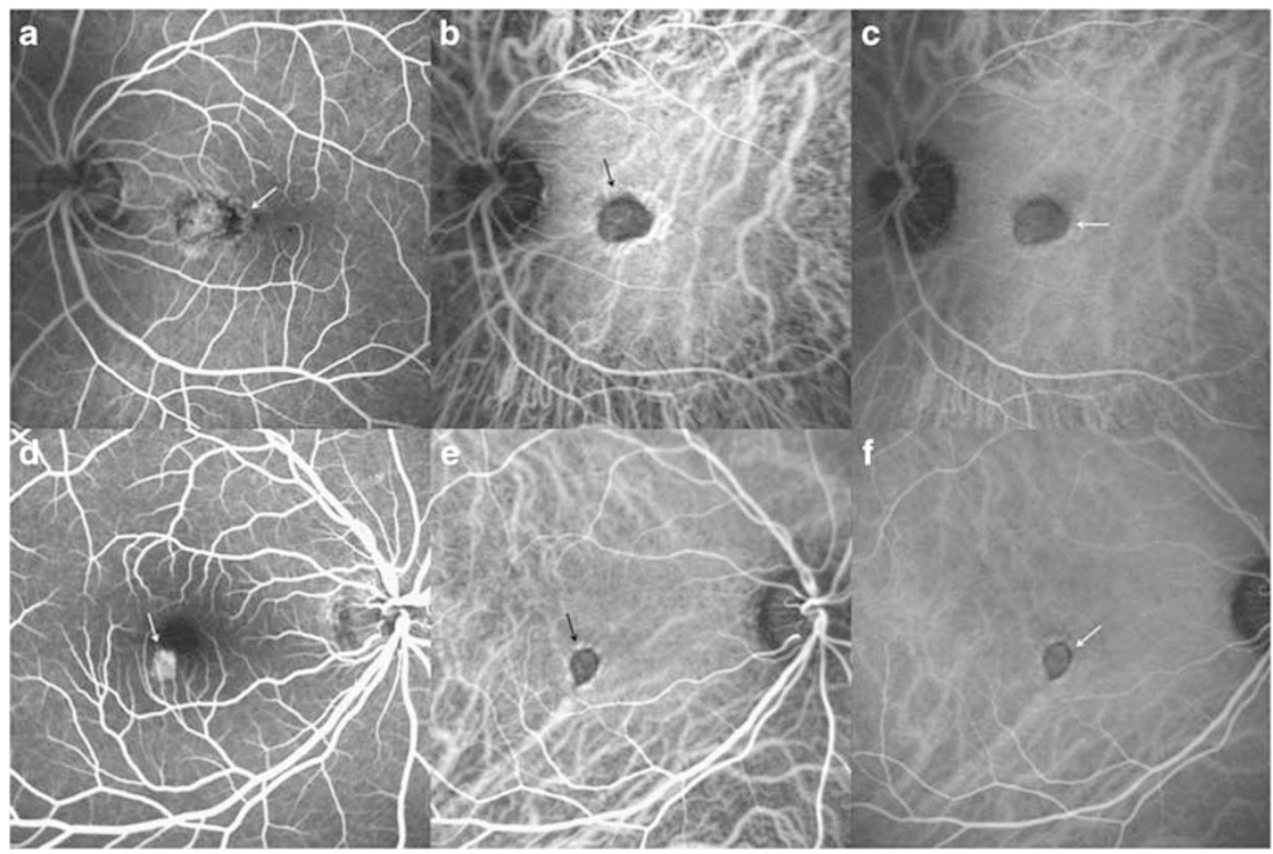

Figure 1 Patients with myopic CNV but without LCs. FA shows fluorescein dye leakage from myopic CNV (white arrow; a and d). Early phase ICGA indicates a dark rim (black arrow; $b$ and e) and late phase ICGA shows a residual dark rim around the neovascular membrane (white arrow; $c$ and $\mathrm{f}$ ).

the side of an existing LC (branching, $26.7 \%$ ), or remote from an existing LC but later connecting with the LC (bridging, 46.7\%; Figures 4 and 5). The progression pattern often appeared as a mixture of these three types. LCs of fellow eyes with no treatment showed $50 \%$ elongation, $33.3 \%$ branching, and 50\% bridging pattern. Conversion of a linear-type LC to the stellate type occurred in one patient (Figure 4).

\section{The relationship between $L C$ and recurrence of myopic CNV}

The development of myopic CNV was detected in 18 treated eyes and 3 fellow eyes. Myopic $\mathrm{CNV}$ recurred at the same location as that of primary $\mathrm{CNV}$ in 7 eyes and at a different location in 11. In all seven eyes in which recurrence was noted in the location of the primary $\mathrm{CNV}$, LCs showed no definite progression. Among the 11 eyes in which recurrence was at different locations, 5 showed progression of LCs, as shown in Figure 6. In six eyes, all with stellate-type LCs, changes in or progression of LCs could not be confirmed. In these cases, small LC fragments were difficult to observe because multiple choroidal atrophy was present and ICGA data were thus of poor quality. The fact that ICGA examinations were conducted at irregular intervals made it difficult to precisely define LC progression.
Three non-CNV fellow eyes showed new development of myopic CNV, with progression of LCs. Two patients complained of metamorphopsia before recurrence of myopic CNV. There was no definite evidence of myopic CNV by fundus photography, FA or ICGA at initial examination, but the condition developed several months later.

\section{Factors predicting recurrence of myopic CNV}

The mean follow-up period for myopic CNV patients was $22.7 \pm 10.1$ months. The follow-up period for patients treated by anti-VEGF injection alone was only 15.9 months, thus shorter than that for other patients receiving PDT only, or the combination treatment who experienced follow-up periods of 27.5 or 26.3 months. The overall recurrence rate of myopic CNV was $36.4 \%$ (24 eyes). In patients treated with PDT only, the overall recurrence rate was $54.5 \%$ (12 eyes), being $50.0 \%$ (9 eyes) and $11.5 \%$ ( 3 eyes) in patients treated with combination therapy and anti-VEGF injection, respectively.

Table 4 shows variables significant in the prediction of myopic CNV recurrence, as identified by Cox's regression analysis. Only the absence of a dark rim showed a significant correlation with recurrence of myopic CNV (odds ratio: 8.05; 95\% confidence interval: 1.65-39.19; $P=0.01$ ). 


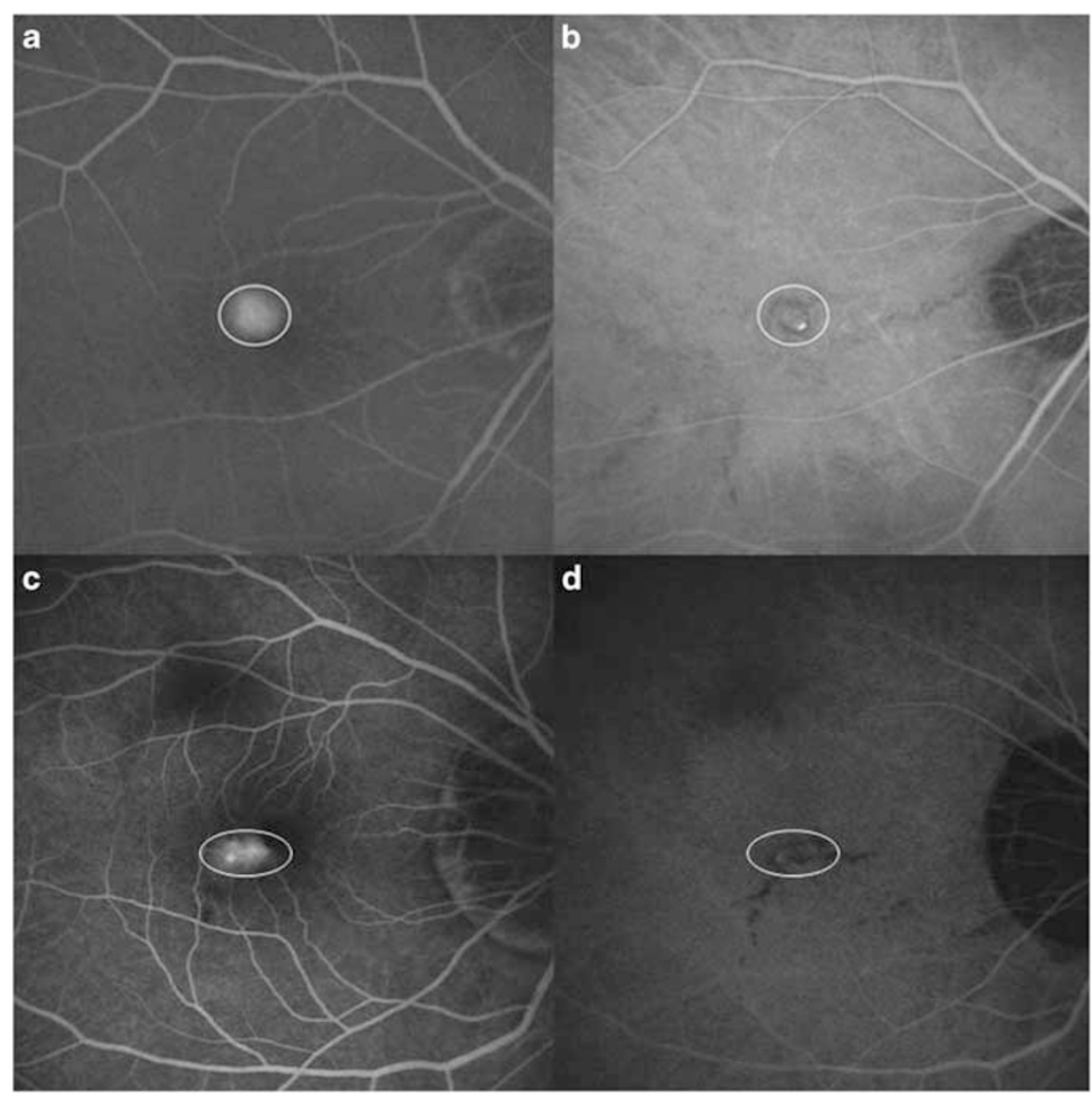

Figure 2 Patients with myopic CNV associated with linear-type LCs. FA shows fluorescein dye leakage (white circle) from myopic CNV ( $a$ and $c$ ). Late phase ICGA indicates small fragments of LCs (white circle; $b$ and $d$ ). The area surrounded by these small crack fragments is the location of myopic CNV. The white circle indicates an identical region on both FA and ICGA.

\section{Discussion}

The characteristic findings of myopic CNV on ICGA, including LC, dark rim, and peripapillary choroidal atrophy, have been used for diagnosis of the condition, and in attempts to understand the mechanism of CNV development. In particular, LCs are formed by ruptures in Bruch's membrane, in which small hemorrhages may develop, and often accompany myopic CNV. Recently, LCs have been considered to induce the development of myopic CNV. ${ }^{13,17,18}$ In this study, we have shown a strong correlation between presence of LC and a relatively large ( $\geq 3000 \mu \mathrm{m}$ ) extent of peripapillary choroidal atrophy.

Few reports have addressed the mechanism of peripapillary choroidal atrophy. Yasuzumi et $a l^{19}$ suggested that a peripapillary myopic crescent might develop as a result of mechanical stretching. If both peripapillary choroidal atrophy and LCs are attributable to stretching of RPE/choroidal tissue induced by myopic axial elongation, these conditions should be of similar prevalence in patients with degenerative myopic fundi.
LCs can be classified in many ways, but are usually divided into two major morphological types: linear and stellate. ${ }^{18,20-22}$ In this study, linear-type LC was evident in 22 eyes, and stellate-type in 15 eyes. Conversion of an LC from the linear type to the stellate type occurred in one patient who had undergone previous PDT treatment, suggesting that PDT may affect the normal growth or progression of an LC.

Among 37 eyes with myopic CNV associated with LCs, CNV had developed along the LCs in 36. In both LC types, each LC consisted of a collection of small dotshaped crack fragments. In linear-type LC, the location of $\mathrm{CNV}$ was in the LC region nearest to the fovea. More precisely, the area of origin was surrounded by small fragments of the LC in a region into which the LC did not extend. The occurred site of $\mathrm{CNV}$ in the center or at the tip of linear-type LC depended on the location of LC with respect to the fovea. When the LC lay over the fovea, $\mathrm{CNV}$ usually developed in the center. However, CNV occurred at the tip of the LC nearest to the fovea when the LC lay outside of the fovea. With stellate-type LCs, 


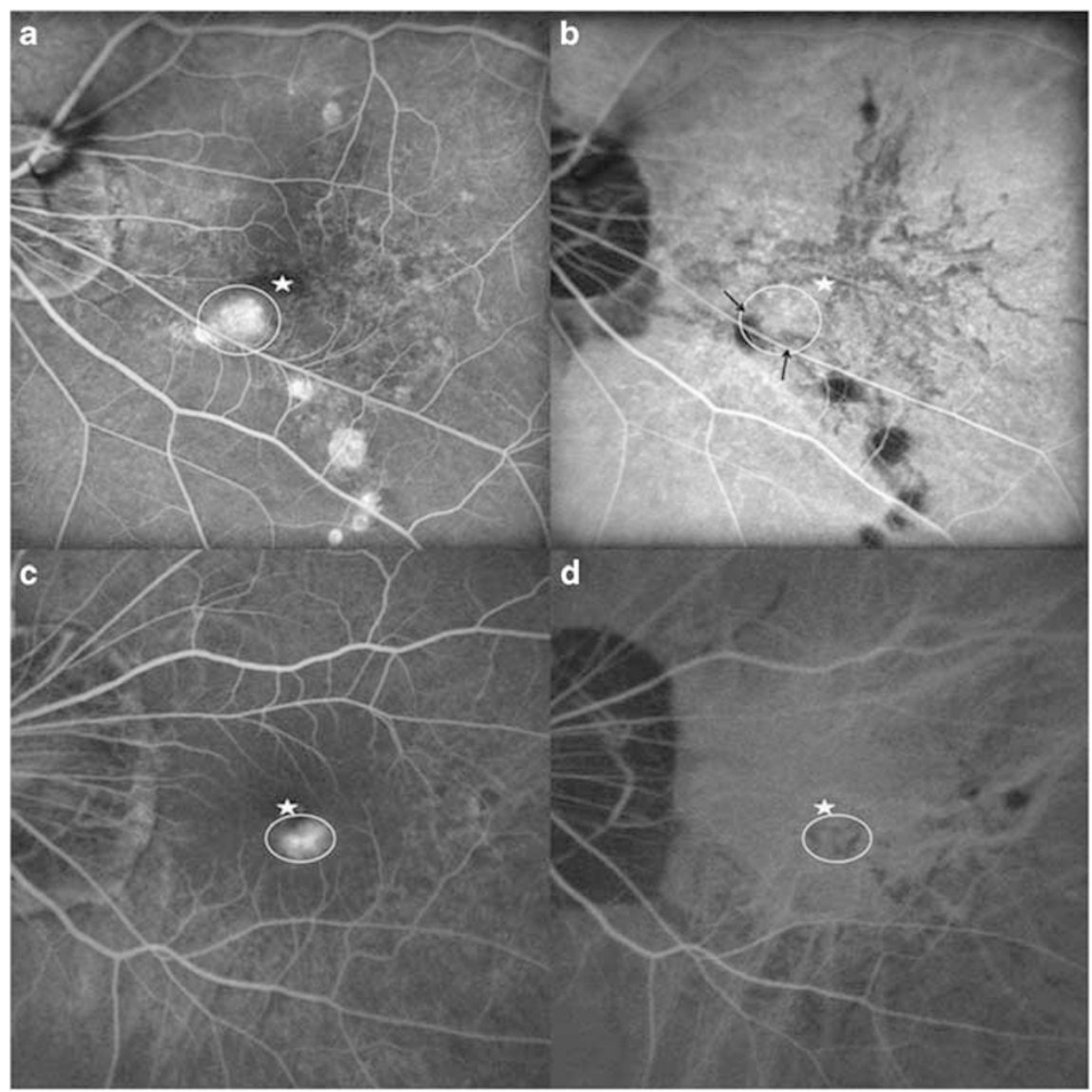

Figure 3 Patients with myopic CNV associated with stellate-type LCs. FA shows fluorescein dye leakage (white circle) from myopic $\mathrm{CNV}$ near the fovea (white star) in two patients (a and c). Myopic CNV is located in the inner corner of the arm radiating from the LC nearest the fovea and small linear-type LCs (black arrows) surrounding CNV are seen close to the fovea (b). Myopic CNV occurred at the tip of a radiating arm near the fovea, whereas a diffuse LC is located at the inferotemporal macula (d).

Table 3 Logistic regression analysis for presence of lacquer crack

\begin{tabular}{|c|c|c|c|c|}
\hline \multirow[t]{2}{*}{ Variables } & \multicolumn{2}{|l|}{ Univariate } & \multicolumn{2}{|l|}{ Multivariate } \\
\hline & Regression coefficient ${ }^{\text {a }}(S E)$ & P-value & Regression coefficient ${ }^{\mathrm{a}}(\mathrm{SE})$ & P-value \\
\hline Age & $-0.202(0.050)$ & $<0.001$ & $-0.260(0.154)$ & 0.09 \\
\hline Gender (female) & $-0.711(0.648)$ & 0.27 & & \\
\hline Refractive error (diopters) & $-0.405(0.120)$ & $<0.001$ & $-0.471(0.462)$ & 0.31 \\
\hline GLD of CNV $(\mu \mathrm{m})$ & $-0.450(0.047)$ & 0.34 & & \\
\hline Presence of dark rim & $-1.699(0.544)$ & 0.002 & $0.924(0.878)$ & 0.62 \\
\hline Size of peripapillary choroidal atrophy $(\mu \mathrm{m})$ & $-0.520(0.132)$ & $<0.001$ & $-0.732(0.329)$ & 0.02 \\
\hline
\end{tabular}

Abbreviations: CNV, choroidal neovascularization; GLD, greatest linear dimension.

${ }^{a}$ The standardized regression coefficient $(\beta)$ was derived for standardization between variables.

the location of CNV was in the inner corner of the radiating arm nearest to the fovea. Detailed imaging and analysis of LCs were possible in this study because of the high sensitivity of the confocal ICGA system.

We categorized the LC progression into three different forms: elongation, branching, and bridging pattern. Most LCs progressed by elongation, but development of myopic CNV was associated with only the branching
(57.1\%) and bridging (42.9\%) patterns. It is possible that some bridging patterns were scored as elongation patterns because of the relatively long intervals between ICGA examinations. Also, some irregular progression patterns could not be categorized using any of the three pattern types. Further studies using regular ICGA examination at short time intervals would be useful to increase our understanding of LC progression. 


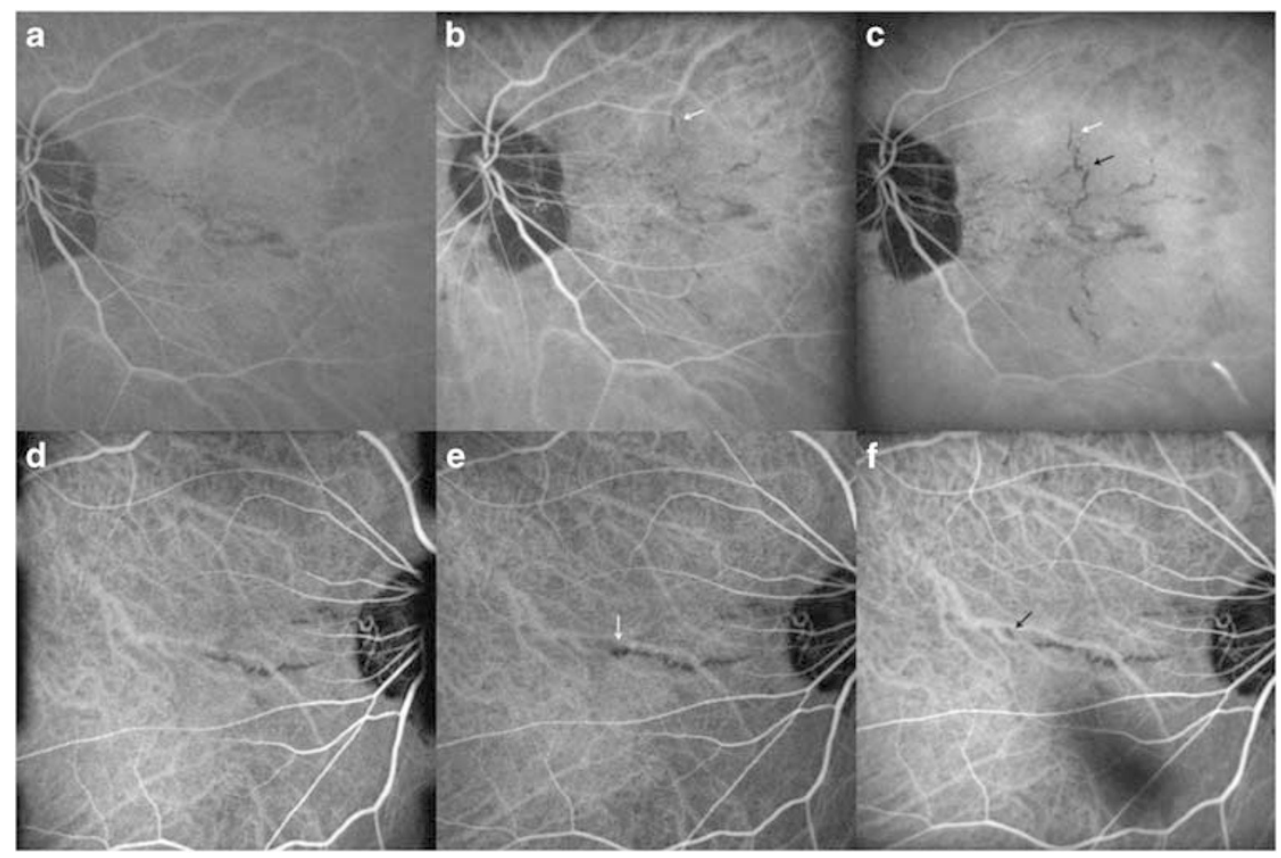

Figure 4 Patients showing LC progression. A linear-type LC is shown on late-phase ICGA (a). At 26 months later, a new small crack, showing a bridging pattern, developed remote from the previous LC (white arrow; b). At 6 months later, another new crack (black arrow) bridged over to the first LC, progressing toward the crack that developed at 26 months (white arrow; c). A linear LC is horizontally located, from the disc to the fovea (d). This LC became extended from a tip with an elongation pattern (white arrow; e). A new crack appeared, remote from the pre-existing LC in a bridging pattern (black arrow; f).

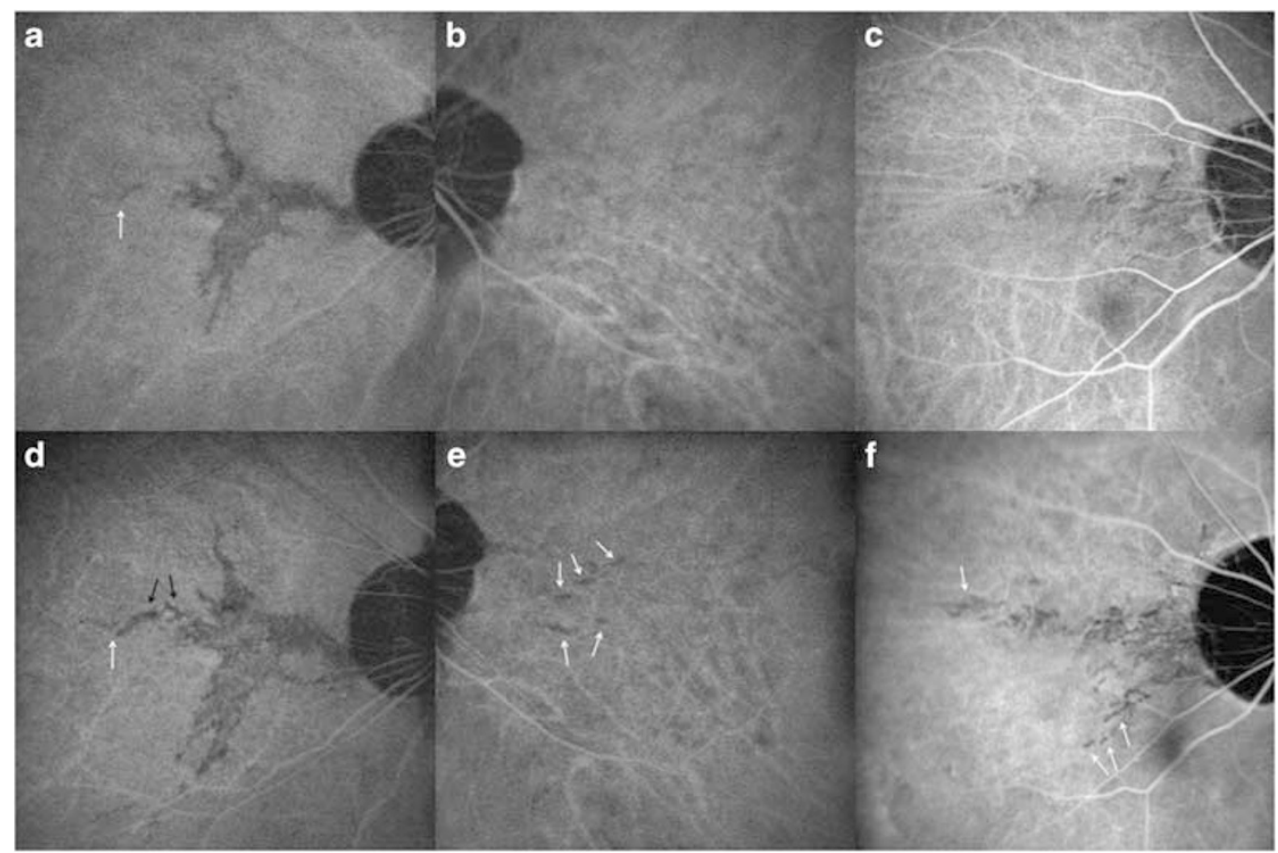

Figure 5 Patients showing LC progression. A stellate-type LC is evident near the disc, extending to the fovea, and small crack fragment in a bridging pattern lies separate from the main LC (white arrow; a). At 21 months later, newly developed LCs (black arrows) bridged the pre-existing LCs (white arrow; d). No definite LC was noted at first (b). At 13 months later, multiple new crack fragments appeared (white arrows; e). Irregular, multiple LCs lie near the disc (c). At 18 months later, new crack fragments in an elongation pattern are evident (white arrows; f). 


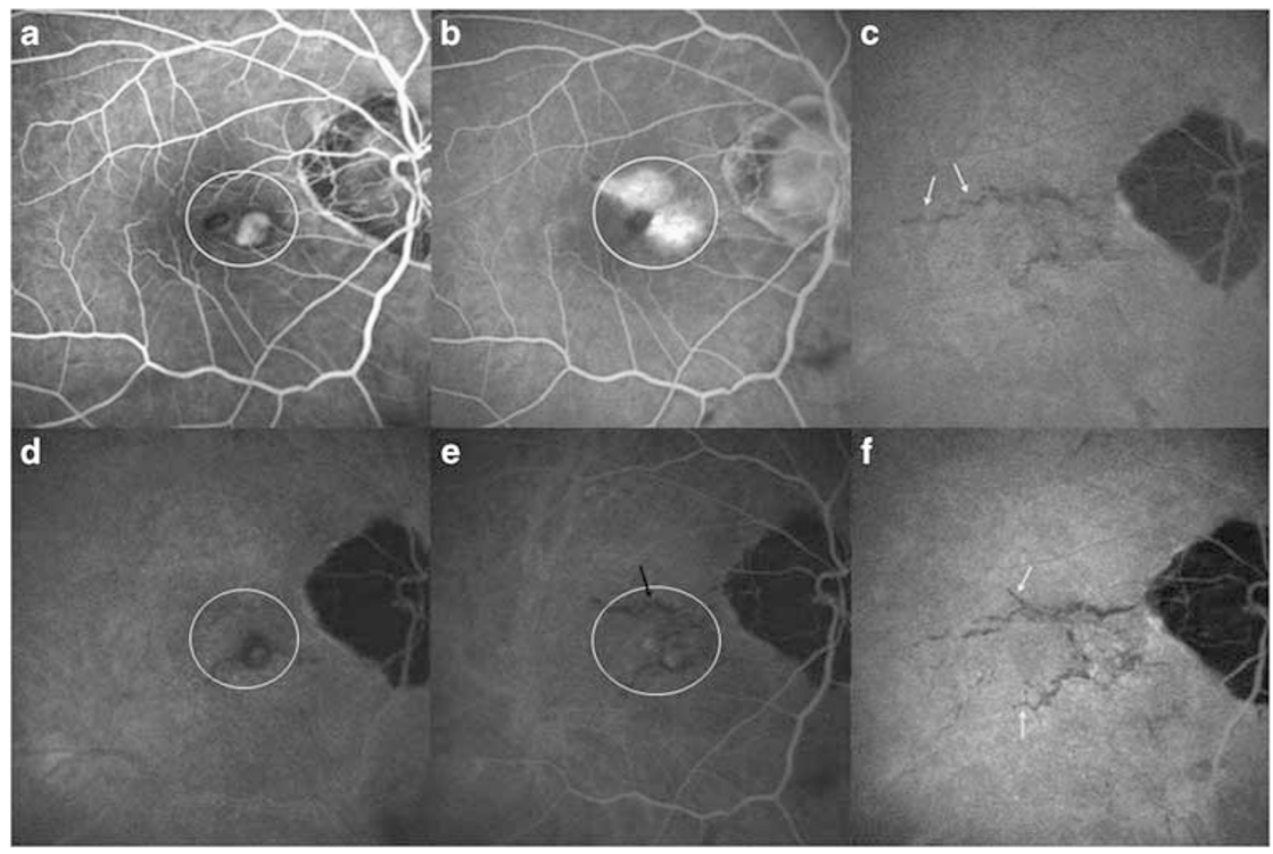

Figure 6 Patients showing LC progression and recurring myopic CNV. FA shows fluorescein dye leakage (white circle) from myopic CNV (a) and late phase ICGA demonstrates an LC surrounding CNV (d). PDT was performed twice and myopic CNV recurred at 9 months later. Fluorescein dye leakages are apparent, from prior and newly developed CNV (b). ICGA reveals an area surrounded by new small crack fragments in a branching pattern (black arrow) that corresponds to CNV (white circle; e). New crack fragments, showing an elongation pattern, appeared at 6 months later (white arrows; c). These cracks progressed slowly in a branching pattern at 19 months later (white arrows; f).

Table 4 Cox's regression analysis model for prognostic factors in recurrence in myopic CNV

\begin{tabular}{lcccc}
\hline Variables & \multicolumn{2}{c}{ Univariate } & & Multivariate \\
\cline { 2 - 3 } & Odds ratio $(95 \%$ CI) & P-value & Odds ratio (95\% CI) & P-value \\
\hline Age & $1.02(0.99-1.05)$ & 0.27 & $0.99(0.97-1.03)$ \\
Gender (female) & $1.60(0.47-5.52)$ & 0.45 & $0.39(0.08-1.92)$ \\
Refractive error (diopters) & $1.01(0.94-1.08)$ & 0.85 & 0.92 \\
GLD of CNV ( $\mu$ m) & $1.00(1.00-1.01)$ & 0.54 & \\
Presence of lacquer crack & $1.08(0.45-2.58)$ & 0.87 & \\
Absence of dark rim & $4.86(1.42-16.66)$ & 0.01 & \\
Treatment of PDT & $0.95(0.27-3.36)$ & 0.94 & \\
Peripapillary choroidal atrophy size $(\mu \mathrm{m})$ & $1.00(1.00-1.00)$ & 0.42 & \\
\hline
\end{tabular}

Abbreviations: CI, confidence interval; CNV, choroidal neovascularization; GLD, greatest linear dimension; PDT, photodynamic therapy.

Progression of LC was also observed on the development of myopic CNV. Five treated eyes showed LC progression and recurrence of CNV at newly developed LCs. Progression of LCs and development of new myopic CNV were detected simultaneously in non-CNV fellow eyes. Outbreak of CNV was associated with recently formed LCs. Axer-Siegel et al ${ }^{17}$ reported that extension of LCs accompanied newly developed myopic CNV and that vigorous healing of new LCs might have contributed to the development of $\mathrm{CNV}$. In this study, LCs progressed with an increase in the number of small crack fragments and myopic CNV was usually located in an area surrounded by these fragments. A possible explanation is that the area surrounded by new small crack fragments, as well as the LC per se, is engaged in a vigorous healing process resulting in initiation of myopic CNV.

Multiple Cox's regression analysis showed that the dark rim was the only significant factor predicting non-recurrence of myopic CNV. Histopathologically, the rim reflects proliferation of RPE cells surrounding CNV in the subretinal space. ${ }^{23}$ The RPE cells block fluorescence of the choroid, and may prevent active leakage from myopic CNV. The dark rim, composed of multilayered proliferating RPE cells surrounding the neovascular membrane, may have a strong regenerative 
potential. Thus, patients showing a dark rim may benefit more from treatments such as PDT or anti-VEGF injections than patients without such a rim. Yoshida et $a l^{24}$ reported that younger myopic CNV patients ( $\leq 40$ years of age) retained relatively good visual acuity without any treatment. However, the age of the patient was not associated with recurrence of myopic CNV in this study.

All patients enrolled in our study had received either PDT and/or anti-VEGF injections to treat myopic CNV in one eye. Thus, LC progression and any relationship thereof with recurrence of myopic CNV might have been affected by these previous treatments. However, non-CNV fellow eyes without myopic CNV were also included in the analysis, and both the pattern of LC progression and the occurrence of myopic CNV were similar.

This study has some limitations, especially in that our data do not precisely reflect the natural course of LC development or recurrence of CNV. Follow-up periods differed between different treatment groups. Eyes receiving PDT or the combined treatment were followed-up for a longer time than were eyes treated by anti-VEGF injection only, because PDT had been performed prior to the more recently available anti-VEGF injection treatment. However, the use of PDT did not influence the recurrence rate of myopic CNV after adjustment of variables to remove covariate bias.

The detailed relationship between LCs and development of myopic CNV, using HRA2 has been described. The results of this study would contribute to an understanding of the pathogenesis and prognosis of myopic $\mathrm{CNV}$, aspects of the condition poorly understood to date.

\section{Summary}

\section{What was known before}

- The characteristic findings of myopic choroidal neovascularization $(\mathrm{CNV})$ on indocyanine green angiography (ICGA), including lacquer crack (LC), dark rim, and peripapillary choroidal atrophy have been used for the diagnosis of this condition and in attempts to understand the mechanism. In particular, LCs are formed by ruptures in Bruch's membrane and often accompany myopic CNV. Recently, LCs have been considered to induce the development of myopic CNV.

\section{What this study adds}

- In this paper, we retrospectively reviewed myopic CNV patients to understand the relationship between lacquer crack and the development of $\mathrm{CNV}$, and to describe the factors prognostic for the recurrence of the condition. This manuscript is unique in that it is a first attempt to analyze ICGA findings of myopic CNV in a relatively large population using Heidelberg retina angiograph 2, confocal laser scanning system that performs fluorescein angiography and ICGA simultaneously.

\section{Conflict of interest}

The authors declare no conflict of interest.

\section{References}

1 Iwase A, Araie M, Tomidokoro A, Yamamoto T, Shimizu H, Kitazawa Y, Tajimi Study Group. Prevalence and causes of low vision and blindness in a Japanese adult population: the Tajimi Study. Ophthalmology 2006; 113: 1354-1362.

2 Cotter SA, Varma R, Ying-Lai M, Azen Sp, Klein R, Los Angeles Latino Eye Study Group. Causes of low vision and blindness in adult Latinos: the Los Angeles Latino Eye Study. Ophthalmology 2006; 113: 1574-1582.

3 Lin LL, Shih YF, Hsiao CK, Chen CJ. Prevalence of myopia in Taiwanese schoolchildren: 1983 to 2000. Ann Acad Med Singapore 2004; 33: 27-33.

4 Wong TY, Foster PJ, Hee J, Ng TP, Tielsch JM, Chew SJ et al. Prevalence and risk factors for refractive errors in adult Chinese in Singapore. Invest Ophthalmol Vis Sci 2000; 41: 2486-2494.

5 Hofman A, Grobbee DE, de Jong PT, van den Ouweland FA. Determinants of disease and disability in the elderly: the Rotterdam Elderly Study. Eur J Epidemiol 1991; 7: 403-422.

6 Wang Q, Klein BE, Klein R, Moss SE. Refractive status in the Beaver Dam Eye Study. Invest Ophthalmol Vis Sci 1994; 35: 4344-4347.

7 Attebo K, Ivers RQ, Mitchell P. Refractive errors in an older population: the Blue Mountains Eye Study. Ophthalmology 1999; 106: 1066-1072.

8 Curtin BJ, Karlin DB. Axial length measurements and fundus changes in the myopic eye. Am J Ophthalmol 1971; 71: $42-50$.

9 Noble KG, Carr RE. Pathologic myopia. Ophthalmology 1982; 89: 1099-1100.

10 Avila MP, Weiter JJ, Jalkh AE, Trempe CL, Pruett RC, Schepens CL. Natural history of choroidal neovascularization in degenerative myopia. Ophthalmology 1984; 91: 1573-1581.

11 Hampton GR, Kohen D, Bird AC. Visual prognosis of disciform degeneration in myopia. Ophthalmology 1983; 90: 923-926.

12 Grossniklaus HE, Green WR. Pathologic findings in pathologic myopia. Retina 1992; 12: 127-133.

13 Ohno-Matsui K, Yoshida T, Futagami S, Yasuzumi K, Shimada N, Kojima A et al. Patchy atrophy and lacquer cracks predispose to the development of choroidal neovascularisation in pathological myopia. $\mathrm{Br} J$ Ophthalmol 2003; 87: 570-573.

14 Quaranta M, Arnold J, Coscas G, Français C, Quentel G, Kuhn $\mathrm{D}$ et al. Indocyanine green angiographic features of pathologic myopia. Am J Ophthalmol 1996; 122: 663-671.

15 Brancato R, Trabucchi G, Introini U, Avanza P, Pece A. Indocyanine green angiography (ICGA) in pathological myopia. Eur J Ophthalmol 1996; 6: 39-43.

16 Ohno-Matsui K, Morishima N, Ito M, Tokoro T. Indocyanine green angiographic findings of lacquer cracks in pathologic myopia. Jpn J Ophthalmol 1998; 42: 293-299.

17 Axer-Siegel R, Cotlear D, Priel E, Rosenblatt I, Snir M, Weinberger D. Indocyanine green angiography in high myopia. Ophthalmic Surg Lasers Imaging 2004; 35: 139-145. 
18 Ikuno Y, Sayanagi K, Soga K, Sawa M, Gomi F, Tsujikawa M et al. Lacquer crack formation and choroidal neovsacularization in pathologic myopia. Retina 2008; 28: 1124-1131.

19 Yasuzumi K, Ohno-Matsui K, Yoshida T, Kojima A, Shimada N, Futagami $\mathrm{S}$ et al. Peripapillary crescent enlargement in highly myopic eyes evaluated by fluorescein and indocyanine green angiography. Br J Ophthalmol 2003; 87: 1088-1090.

20 Soubrane G, Coscas GJ. Choroidal neovascular membrane in degenerative myopia. In: Ryan SJ (ed). Retina, 4th edn. Mosby: St Louis, MO, 2005; 1136-1152.

21 Ladas ID, Moschos MM, Rouvas AA, Karagiannis DA, Kokolakis SN. Lacquer crack formation after photodynamic therapy. Eur J Ophthalmol 2003; 13: 729-733.
22 Johnson DA, Yannuzzi LA, Shakin JL, Lightman DA. Lacquer cracks following laser treatment of choroidal neovascularization in pathologic myopia. Retina 1998; 18: 118-124.

23 Fukushima I, Takahashi K, Nishimura T, Ohkuma H, Uyama M. Dark rim around choroidal neovascularization in indocyanine green angiography. Nippon Ganka Gakkai Zasshi 1995; 99: 1262-1270.

24 Yoshida T, Ohno-matsui K, Ohtake Y, Takashima T, Futagami S, Baba T et al. Long-term visual prognosis of choroidal neovascularization in high myopia: a comparison between age groups. Ophthalmology 2002; 109: $712-719$. 